\title{
Vertragsrecht in der Corona-Krise \\ COVInsAG: Auswirkungen auf die Insolvenzantragspflicht und die Haftung der Organe
}

\author{
Prof. Dr. Jens M. Schmittmann, Essen*
}

\section{Inhaltsübersicht}

A. Einleitung 246

B. Umfeld des Gesetzes und Gesetzgebungsverfahren 246

C. Das COVID-19-Insolvenzaussetzungsgesetz 247

I. Aussetzung der Insolvenzantragspflicht 247

II. Folgen der Aussetzung der Insolvenzantragspflicht 248

1. Vereinbarkeit von Zahlungen mit der Sorgfalt eines ordentlichen
Geschäftsleiters

2. Privilegierung der Rückzahlung von Darlehen 249

3. Ausschluss der Sittenwidrigkeit der Kreditgewährungen 249

4. Ausschluss der Insolvenzanfechtung 249

5. Erweiterung des Anwendungsbereichs 249

III. Gläubigeranträge $\quad 250$

IV. Verordnungsermächtigung 250

D. Die Massesicherungspflicht in Zeiten der Corona-Krise $\quad 251$

I. Grundlagen der Massesicherungspflicht 251

II. Modifikation durch $\$ 2$ Abs. 1 Nr. 1 COVInsAG 251

1. Ersatzanspruch bei Scheitern der Sanierung 252

2. Insolvenzgründe und deren Feststellung 252

3. Reichweite der Massesicherungsplicht 253

4. Geltendmachung durch den Insolvenzverwalter 254

III. Wechselwirkungen zum Insolvenzanfechtungsrecht 255

IV. Wechselwirkungen zum Haftungs- und Deliktsrecht 255

E. Fazit und Ausblick 256

* Der Verfasser ist Professor für Allgemeine Betriebswirtschaftslehre, Wirtschaftsund Steuerrecht an der FOM Hochschule für Oekonomie und Management Essen, Rechtsanwalt, Fachanwalt für Insolvenzrecht, für Handels- und Gesellschaftsrecht und Steuerrecht sowie Steuerberater und Mitglied des Senats für Anwaltssachen des Bundesgerichtshofs. Darüber hinaus ist er Vizepräsident des RIFAM RheinRuhr-Instituts für angewandte Mittelstandsforschung e.V., Düsseldorf. 


\section{A. Einleitung}

Die Ausbreitung des SARS-CoV-2-Virus weltweit und in Deutschland, die auch als „COVID-19-Pandemie“ bezeichnet wird, beschränkt derzeit das öffentliche Leben massiv. Freizeit- und Kultureinrichtungen werden ebenso geschlossen wie Kinderbetreuungseinrichtungen, Gastronomiebetriebe und Einzelhandelsgeschäfte. Darüber hinaus werden viele öffentliche Veranstaltungen abgesagt. All diese Umstände führen dazu, dass Personen, die ihren Lebensunterhalt in diesem Bereich erwirtschaften, Einkommensverluste erleiden. Zudem ist bei vielen Unternehmen Kurzarbeit angeordnet, was ebenfalls zu wirtschaftlichen Einbußen der Arbeitnehmer führt. Unternehmen erleiden massive Umsatzeinbrüche und geraten schnell in eine Liquiditätskrise.

\section{B. Umfeld des Gesetzes und Gesetzgebungsverfahren}

Der Gesetzgeber hat früh Maßnahmen ergriffen. Schon am 13. März 2020 ist das Gesetz zur befristeten krisenbedingten Verbesserung der Regelungen für das Kurzarbeitergeld beschlossen worden. Es folgten dann innerhalb weniger Tage das Gesetz über die Feststellungen eines Nachtrages zum Bundeshaushaltsplan für das Jahr 2020 („Nachtragshaushaltsgesetz $\left.2020^{\circ}\right)$, das Gesetz für den erleichterten Zugang zu sozialer Sicherung und zum Einsatz und zur Absicherung sozialer Dienstleister auf Grund des Coronavirus SARS-CoV-2 („Sozialschutzpaket“), das Gesetz zum Ausgleich COVID-19 bedingter finanzieller Belastungen der Krankenhäuser und weiterer Gesundheitseinrichtungen („COVID-19-Krankenhausentlastungsgesetz“) sowie das Gesetz zum Schutz der Bevölkerung bei einer epidemischen Lage von nationaler Tragweite, die sämtlich am 27. März 2020 beschlossen und im Bundesgesetzblatt verkündet worden sind.

Im Rahmen dieser Gesetzgebungsverfahren wurde auch das Gesetz zur vorübergehenden Aussetzung der Insolvenzantragspflicht und zur Begrenzung der Organhaftung bei einer durch die COVID-19-Pandemie bedingten Insolvenz („COVInsAG“) als Art. 1 des Gesetzes zur Abmilderung der 
Folgen der COVID-19-Pandemie im Zivil-, Insolvenz- und Strafverfahrensrecht vom 27. März $2020^{1}$ verabschiedet und verkündet.

Das Gesetzgebungsverfahren war von großer Eile getrieben. Zwischen der Formulierungshilfe der Bundesregierung vom 20. März 2020, 21:12 Uhr, der Kabinettsvorlage vom 22. März 2020, dem Gesetzentwurf der Fraktionen der CDU/CSU und SPD vom 24. März 2020 und der Beschlussfassung des Bundesrates sowie der Verkündung im Bundesgesetzblatt am 27. März 2020 lagen nur wenige Tage.

\section{Das COVID-19-Insolvenzaussetzungsgesetz}

\section{Aussetzung der Insolvenzantragspflicht}

Kern des COVInsAG ist die Aussetzung der Insolvenzantragspflicht. Nach $\$ 1$ COVInsAG ist die Pflicht zur Stellung eines Insolvenzantrages nach

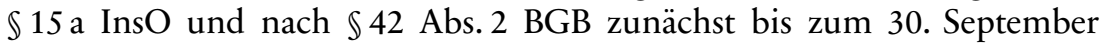
2020 ausgesetzt.

Es handelt sich - so jedenfalls die eindeutige Formulierung - um eine allgemeine Aussetzung der Insolvenzantragspflicht, die lediglich dann nicht eingreift, wenn die Insolvenzreife nicht auf den Folgen des SARSCOV-2-Virus (COVID-19-Pandemie) beruht, oder wenn keine Aussichten darauf bestehen, eine bestehende Zahlungsunfähigkeit zu beseitigen.

Weiterhin gilt die Vermutung, dass die Insolvenzreife auf den Auswirkungen der COVID-19-Pandemie beruht und Aussichten darauf bestehen, eine bestehende Zahlungsunfähigkeit zu beseitigen, wenn der Schuldner am 31. Dezember 2019 nicht zahlungsunfähig war. Da die Überschuldung im Wortlaut des $₫ 1$ COVInsAG nicht genannt ist, steht sie der Anwendung der Vermutung nicht entgegen.

Auch wenn für natürliche Personen keine Insolvenzpflicht besteht, so sind sie gleichwohl zur Vermeidung einer Versagung der Restschuldbefreiung im Schlusstermin gehalten, einen Insolvenzantrag zu stellen. Die Restschuldbefreiung ist nämlich gem. $\$ 290$ Abs. $1 \mathrm{Nr} .4$ InsO zu versagen, wenn der Schuldner in den letzten drei Jahren vor dem Antrag auf Eröffnung des Insolvenzantrages oder nach dem Antrag vorsätzlich oder grob fahrlässig die Befriedigung der Insolvenzgläubiger dadurch beeinträchtigt hat, dass er unangemessene Verbindlichkeiten begründet oder Vermögen

1 BGBl. I 2020, 569 ff. Vgl. dazu: G. Pape, Temporäre Aussetzung der Insolvenzantragspflicht in der Corona-Krise, NWB 2020, $1053 \mathrm{ff}$. 
verschwendet oder ohne Aussicht auf eine Besserung seiner wirtschaftlichen Lage die Eröffnung des Insolvenzverfahrens verzögert. Dadurch soll verhindert werden, dass Schuldner kurz vor Insolvenzantragstellung ihr Vermögen verprassen ${ }^{2}$. Im Hinblick auf die vielen Einzelgewerbetreibenden und sonstigen Selbständigen, die durch die COVID-19-Pandemie in wirtschaftliche Schwierigkeiten geraten, wird $\$ 290$ Abs. 1 Nr. 4 InsO dahin modifiziert, dass auf die Verzögerung der Eröffnung des Insolvenzverfahrens im Zeitraum zwischen dem 1. März 2020 und dem 30. September 2020 keine Versagung der Restschuldbefreiung gestützt werden kann.

Die Strafbarkeit wegen Insolvenzverschleppung ist auf Grund der Aussetzung der Insolvenzantragsplicht bei Vorliegen der Voraussetzungen gem. $\$ 1$ COVInsAG aufgehoben. Es bleibt allerdings weiter möglich, die Straftaten des Eingehungsbetruges gem. $\$ 263$ StGB, der Nichtabführung des Arbeitnehmeranteils des Sozialversicherungsbeitrags gem. $\$ 266$ a StGB oder des Bankrotts gem. $₫ 283$ BGB zu begehen ${ }^{3}$.

\section{Folgen der Aussetzung der Insolvenzantragspflicht}

Die Folgen der Aussetzung der Insolvenzantragspflicht gem. $₫ 1$ COVInsAG sind in $\$ 2$ COVInsAG geregelt ${ }^{4}$.

\section{Vereinbarkeit von Zablungen mit der Sorgfalt eines ordentlichen Geschäfsleiters}

Soweit die Insolvenzantragspflicht ausgesetzt ist, gelten gem. $\$ 2$ Abs. 1 Nr. 1 COVInsAG Zahlungen, die im ordnungsgemäßen Geschäftsgang erfolgen, insbesondere solche Zahlungen, die der Aufrechterhaltung oder Wiederaufnahme des Geschäftsbetriebes oder der Umsetzung eines Sanie-

2 Vgl. J. M. Schmittmann/H. Theurich/T. Brune, Das insolvenzrechtliche Mandat, 5. Auflage, Bonn, 2017, $\$ 6$ Rdnr. 260.

3 S. R. Theiselmann/A. Verhoeven, Die Aussetzung der Insolvenzantragspflicht und Anpassung des Zahlungsverbots aus Sicht der Geschäftsleiter von Lieferanten, ZIP 2020, 797, 799.

4 Vgl. Ch. Thole, Die Aussetzung der Insolvenzantragspflicht nach dem COVID-19Insolvenz-Aussetzungsgesetz und ihre weiteren Folgen, ZIP 2020, 650 (654); J. M. Schmittmann, Die insolvenzrechtliche Komponente des Gesetzes zur Abmilderung der Folgen der COVID-19-Pandemie im Zivil-, Insolvenz- und Strafverfahrensrecht vom 27. März 2020, ZRI 2020, 234 (236). 
rungskonzeptes dienen, als mit der Sorgfalt eines ordentlichen und gewissenhaften Geschäftsleiters i. S. d. $\$ 64$ Satz 2 GmbHG, des $\$ 92$ Abs. 2 Satz 2 AktG, des $₫ 130$ a Abs. 1 Satz 2 HGB, auch i. V. m. $\$ 177$ a Satz 1 HGB und des $₫ 99$ Satz 2 GenG vereinbar.

\section{Privilegierung der Rückzablung von Darlehen}

Darüber hinaus gilt gem. $\ 2$ Abs. 1 Nr. 2 COVInsAG die bis zum 30. September 2023 erfolgende Rückgewähr eines im Aussetzungszeitraum gewährten neuen Kredites sowie die im Aussetzungszeitraum erfolgte Bestellung von Sicherheiten zur Absicherung solcher Kredite als nicht gläubigerbenachteiligend. Auch die Rückgewähr von Gesellschafterdarlehen ist nicht anfechtbar, sondern lediglich die Besicherung.

\section{Ausschluss der Sittenwidrigkeit der Kreditgewährungen}

Gem. $\$ 2$ Abs. 1 Nr. 3 COVInsAG sind Kreditgewährungen und -besicherungen im Aussetzungszeitraum nicht als sittenwidriger Beitrag zur Insolvenzverschleppung anzusehen, soweit nach $\$ 1$ COVInsAG die Pflicht zur Stellung eines Insolvenzantrages ausgesetzt ist.

\section{Ausschluss der Insolvenzanfechtung}

Weitreichende Anfechtungsausschlüsse sind Gegenstand von $₫ 2$ Abs. 1 Nr. 4 COVInsAG, wonach kongruente Deckungen von der Anfechtung ebenso ausgeschlossen sind wie bestimmte inkongruente Deckungen. Es bleibt lediglich bei der Möglichkeit der Anfechtung, wenn dem anderen Teil bekannt war, dass die Sanierungs- und Finanzierungsbemühungen des Schuldners nicht zur Beseitigung einer eingetretenen Zahlungsunfähigkeit geeignet gewesen sind.

\section{Erweiterung des Anwendungsbereichs}

Gem. $\$ 2$ Abs. 2 COVInsAG werden diese Regelungen auch auf Unternehmen erstreckt, die keiner Antragspflicht unterliegen, sowie auf Schuldner, die weder zahlungsunfähig noch überschuldet sind. 
Soweit Finanzierungen durch die Kreditanstalt für Wiederaufbau und ihre Finanzierungspartner im Rahmen staatlicher Hilfsprogramme im Rahmen der COVID-19-Pandemie gewährt worden sind, ist die Rückzahlung auch über den 30. September 2023 hinaus nicht anfechtbar.

\section{Gläubigeranträge}

Das Gesetz sieht zudem vor, dass bei zwischen dem 28. März 2020 und dem 28. Juni 2020 gestellten Gläubigerinsolvenzanträgen die Eröffnung des Insolvenzverfahrens gem. $\$ 3$ COVInsAG voraussetzt, dass der Eröffnungsgrund bereits am 1. März 2020 vorlag. Nunmehr wird es zur Vermeidung der Abweisung des Antrags als unzulässig Sache des Gläubigers sein, mit der Antragstellung glaubhaft zu machen, dass der Eröffnungsgrund bereits am 1. März 2020 vorlag, z. B. durch Vorlage von Pfändungsprotokollen, aus denen sich die Zahlungsunfähigkeit ergibt. Je weiter die Zeit fortschreitet, umso schwieriger dürfte es werden, diesen Nachweis zu führen. Dies ist vom Gesetzgeber allerdings auch beabsichtigt, da Eröffnungsanträge der Gläubiger vermieden werden sollen, um die Insolvenzreife von Unternehmen mit Hilfe von Hilfs- und Stabilisierungsmaßnahmen und sonstiger Sanierungs- oder Finanzierungsmaßnahmen zu beseitigen ${ }^{5}$. Gem. Art. 6 Abs. 1 des Gesetzes zur Abmilderung der Folgen der COVID-19-Pandemie im Zivil-, Insolvenz- und Strafverfahrensrecht vom 27. März 2020 ist die Regelung mit Wirkung vom 1. März 2020 in Kraft getreten, so dass die Gerichte in bereits anhängigen Gläubigerantragsverfahren zusätzlich prüfen müssen, wann der Insolvenzgrund eingetreten ist.

\section{Verordnungsermächtigung}

Das Gesetz schließt mit einer Verordnungsermächtigung. Das Bundesministerium für Justiz und für Verbraucherschutz wird ermächtigt, durch Rechtsverordnung ohne Zustimmung des Bundesrates die Aussetzung der Insolvenzantragspflicht nach $\mathbb{1} 1$ COVInsAG und die Regelung zum Eröffnungsgrund bei Gläubigerinsolvenzanträgen nach $₫ 3$ COVInsAG bis höchstens zum 31. März 2021 zu verlängern, wenn dies auf Grund fortbe-

5 So Gesetzentwurf der Fraktionen der CDU/CSU und SPD vom 24. März 2020, BTDrucksache 19/18110, S. 24. 
stehender Nachfrage nach verfügbaren öffentlichen Hilfen, andauernder Finanzierungschwierigkeiten oder sonstiger Umstände geboten scheint.

Ein Verweis auf $₫ 2$ COVInsAG fehlt in $₫ 4$ COVInsAG, ist allerdings auch nicht erforderlich, da $\$ 2$ COVInsAG unmittelbar auf die Aussetzung der Insolvenzantragspflicht in $\$ 1$ COVInsAG verweist.

\section{Die Massesicherungspflicht in Zeiten der Corona-Krise}

Ein wesentlicher Baustein der Massesicherungspflicht ist die Haftung der Organe für Zahlungsabflüsse, die nach Eintritt der materiellen Insolvenzreife erfolgen. Das System ist bei allen haftungsbeschränkten Gesellschaften vergleichbar. Nachfolgend wird das Beispiel der $\mathrm{GmbH}$ verwendet, da es sich bei der $\mathrm{GmbH}$ um die häufigste haftungsbeschränkte Gesellschaftsform handelt. Für die haftungsbeschränkte OHG, die haftungsbeschränkte Kommanditgesellschaft, die Aktiengesellschaft sowie die Genossenschaft gelten die nachstehenden Ausführungen sinngemäß.

\section{Grundlagen der Massesicherungspflicht}

Geschäftsführer sind nach $\$ 64$ Satz 1 GmbHG der Gesellschaft zum Ersatz von Zahlungen verpflichtet, die nach Eintritt der Zahlungsunfähigkeit der Gesellschaft oder nach Feststellung ihrer Überschuldung geleistet werden. Es handelt sich schon dem Wortlaut nach nicht um einen Schadensersatzanspruch, sondern um einen Erstattungsanspruch eigener Art $^{6}$ („sui generis“). Gem. $\$ 64$ Satz 2 GmbHG gilt der Erstattungsanspruch nicht für Zahlungen, die auch nach diesem Zeitpunkt mit der Sorgfalt eines ordentlichen Geschäftsmannes vereinbar sind. Für diese Ausnahme trägt der Geschäftsführer die Darlegungs- und Beweislast.

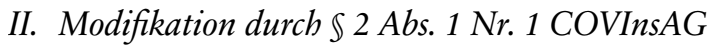

Gem. $\mathbb{2}$ Abs. 1 Nr. 1 COVInsAG gelten Zahlungen, die im ordnungsgemäßen Geschäftsgang erfolgen, insbesondere solche Zahlungen, die der

6 So BGH, Urteil vom 11. Februar 2020 - II ZR 427/18, ZInsO 2020, 779 ff. Rn. 21 (zu $₫ 130$ a Abs. 2 Satz 1 HGB); BGH, Urteil vom 21. Mai 2019 - II ZR 337/17, ZInsO 2019, 1529 = ZIP 2019, 1719 ff. Rn. 16 (zu $\$ 64$ Satz 1 GmbHG). 
Aufrechterhaltung und Wiederaufnahme des Geschäftsbetriebes oder der Umsetzung eines Sanierungskonzeptes dienen, als mit der Sorgfalt eines ordentlichen und gewissenhaften Geschäftsleiters i. S. d. $\$ 64$ Satz 2 GmbHG (und der für die übrigen Gesellschaftsformen geltenden vergleichbaren Vorschriften) vereinbar. Auch wenn der Anspruch gem. $\$ 64$ Satz 1 GmbHG nicht von der Eröffnung des Insolvenzverfahrens abhängt, so wird er in aller Regel lediglich vom Insolvenzverwalter geltend gemacht.

\section{Ersatzanspruch bei Scheitern der Sanierung}

Für den Fall einer gescheiterten Sanierung wird der Insolvenzverwalter daher zunächst festzustellen haben, wann materiell die Insolvenzreife eingetreten ist, also prüfen, wann Zahlungsunfähigkeit $(\mathbb{1} 17 \mathrm{InsO})$ und/oder Überschuldung ( $\$ 19$ InsO) eingetreten sind.

\section{Insolvenzgründe und deren Feststellung}

Von Zahlungsunfähigkeit ist nach der Rechtsprechung des BGH auszugehen, wenn die Liquiditätslücke des Schuldners zehn oder mehr Prozent beträgt, sofern nicht ausnahmsweise mit an Sicherheit grenzender Wahrscheinlichkeit zu erwarten ist, dass die Liquiditätslücke demnächst vollständig oder fast vollständig beseitigt werden wird und den Gläubigern ein Zuwarten nach den besonderen Umständen des Einzelfalles zuzumuten ist. Dazu ist zu prüfen, welche liquiden Mittel in den nächsten drei Wochen zur Verfügung stehen ${ }^{7}$. Bei der Feststellung der Zahlungsunfähigkeit dem. $\mathbb{1} 17$ Abs. 2 Satz 1 InsO anhand einer Liquiditätsbilanz sind auch die innerhalb von drei Wochen fällig werdenden und eingeforderten Verbindlichkeiten (sog. Passiva II) einzubeziehen ${ }^{8}$.

Die Feststellung der Überschuldung stellt sich oftmals noch schwieriger dar. Überschuldung liegt gem. $\mathbb{1} 19$ Abs. 2 Satz 1 InsO vor, wenn das Vermögen des Schuldners die bestehenden Verbindlichkeiten nicht mehr deckt, es sei denn, die Fortführung des Unternehmens ist nach den Um-

7 So BGH, Urteil vom 24. Mai 2005 - IX ZR 123/04, BGHZ 163, 134 ff. = NZI 2005, 547 ff. mit Anm. H. Thonfeld.

8 So BGH, Urteil vom 19. Dezember 2017 - II ZR 88/16, ZIP 2018, 283 ff. = NZI 2018, $204 \mathrm{ff}$. 
ständen überwiegend wahrscheinlich. Trägt der Insolvenzverwalter eine handelsbilanzielle Überschuldung vor, sowie das Fehlen stiller Reserven, geht die sekundäre Darlegungs- und Beweislast auf den Geschäftsführer über ${ }^{9}$. Zur Feststellung einer Überschuldung einer $\mathrm{GmbH}$ bedarf es nach der Rechtsprechung des BGH stets eines Überschuldungsstatus in Form einer Vermögensbilanz, die über die „wahren Werte“ des Gesellschaftsvermögens Auskunft gibt ${ }^{10}$.

\section{Reichweite der Massesicherungspflicht}

Unter Zahlungen gem. $\$ 64$ Satz 1 GmbHG ist jede masseschmälernde Leistung zu verstehen ${ }^{11}$. Der Zahlungsbegriff in $\$ 64$ Satz $1 \mathrm{GmbHG}$ ist weit auszulegen ${ }^{12}$.

Auch der Forderungseinzug auf einem debitorisch geführten Konto kann als Verstoß gegen die Massesicherungspflicht gesehen werden, allerdings nur dann, wenn die Forderung nicht an die Bank zur Sicherheit abgetreten war und wenn die Sicherungsabtretung vor Insolvenzreife vereinbart und die Forderung der Gesellschaft entstanden und werthaltig geworden ist. Zudem liegt auch keine Zahlung i. S. d. $\$ 64$ Satz 1 GmbHG vor, soweit in Folge der Verminderung des Debetsaldos durch die Einziehung und Verrechnung einer Forderung weitere sicherungsabgetretene Forderungen frei werden ${ }^{13}$.

Weiterhin hat BGH zum Entfall der Haftung des Gesellschaftsorgans für Zahlungen nach Insolvenzreife aufgrund Ausgleichs der Masseschmälerung durch Gegenleistung entschieden, dass die Ersatzpflicht in diesen Fällen jedenfalls dann entfällt, wenn der in die Masse gelangende Gegenstand für die Verwertung durch die Gläubiger geeignet ist. Wenn die Gesellschaft insolvenzreif und eine Liquidation zu Grunde zu legen ist, ist die in

9 So BGH, Urteil vom 19. November 2013 - II ZR 229/11, ZIP 2014, 168 ff. = NZI 2014, $233 \mathrm{ff}$.

10 So BGH, Beschluss vom 23. Juli 2005 - 3 StR 518/14, ZInsO 2015, $2021 \mathrm{f}$.

11 So BGH, Urteil vom 28. Februar 2012 - II ZR 244/10, NZI 2012, 569 ff. = AG 2012, 371 ff.; BGH, Urteil vom 16. März 2009 - II ZR 32/08, ZIP 2009, 956 ff. = NZI 2009, $486 \mathrm{ff}$.

12 So BGH, Urteil vom 16. März 2009 - II ZR 32/08, ZIP 2009, 956 ff. = NZI 2009, 286 ff.; vgl. J. M. Schmittmann, Haftung der Organe in Krise und Insolvenz, 2. Auflage, Köln, 2018, Rdnr. 327.

13 So BGH, Urteil vom 23. Juni 2015 - II ZR 366/13, BGHZ 206, 52 ff. = ZIP 2015, $1480 \mathrm{ff}$. 
die Masse gelangende Gegenleistung grundsätzlich nach Liquidationswerten zu bemessen ${ }^{14}$.

Die Haftung des Geschäftsführers ist nicht allein deshalb ausgeschlossen, weil eine Sanierung beabsichtigt ist. Der BGH forderte bislang im Rahmen von $\$ 64$ Satz 2 GmbHG eine konkrete Chance auf Sanierung und Fortführung. Die Sanierungsabsicht allein entschuldige Zahlungen nach Insolvenzreife nicht ${ }^{15}$.

Hinter diesen Anforderungen bleibt die Regelung im COVInsAG zurück. Nach $₫ 2$ Abs. 1 Nr. 1 COVInsAG gelten Zahlungen, die im ordnungsgemäßen Geschäftsgang erfolgen, insbesondere solche Zahlungen, die der Aufrechterhaltung und Wiederaufnahme des Geschäftsbetriebes oder der Umsetzung eines Sanierungskonzeptes dienen, als mit der Sorgfalt eines ordentlichen und gewissenhaften Geschäftsleiters vereinbar. Es ist lediglich noch gefordert, dass die Zahlung der „Umsetzung eines Sanierungskonzeptes" dient. Es muss sich weder um eine konkrete Chance auf Sanierung handeln noch muss das Sanierungskonzept aussichtsreich sein.

\section{Geltendmachung durch den Insolvenzverwalter}

Der Insolvenzverwalter wird im Falle des Scheiterns der Sanierung und der Eröffnung des Insolvenzverfahrens mit Aussicht auf Erfolg einen Anspruch gem. $\$ 64$ Satz 1 GmbHG geltend machen können, wenn ihm der Nachweis gelingt, dass schon keine Aussetzung der Insolvenzantragspflicht gem. $\$ 1$ COVInsAG gegeben war. Dazu könnte der Insolvenzverwalter z. B. vortragen und beweisen, dass der Schuldner bereits vor dem 31. Dezember 2019 zahlungsunfähig war. Weiterhin könnte der Insolvenzverwalter vortragen und beweisen, dass keine Aussichten darauf bestanden, eine bestehende Zahlungsunfähigkeit zu beseitigen. Sofern dem Insolvenzverwalter dieser Nachweis gelingt, ist per se $\mathbb{2}$ Abs. 1 COVInsAG ausgeschlossen.

Ist der Geltungsbereich von $\$ 1$ COVInsAG indes eröffnet, kann der Insolvenzverwalter gleichwohl den Geschäftsführer mit Aussicht auf Erfolg in Anspruch nehmen, wenn es sich um Zahlungen handelte, die nicht der

14 So BGH, Urteil vom 4. Juli 2017 - II ZR 319/15, ZIP 2017, 1619 ff. = NZI 2017, 809 ff. mit Anm. J. Schädlich.

15 So BGH, Beschluss vom 24. September 2019 - II ZR 248/17, NZI 2020, 180 ff. mit Anm. A. J. Baumert = DStR 2020, 179 ff.; BGH, Urteil vom 4. Juli 2017 - II ZR 319/15, ZIP 2017, 1847 ff.; BGH, Beschluss vom 7. Februar 2007 - II ZR 51/06, ZIP 2007, $1501 \mathrm{ff}$. 
Aufrechterhaltung oder Wiederaufnahme des Geschäftsbetriebes dienten. Hierbei dürfte es sich beispielsweise um Zahlungen an Gesellschafter handeln oder auch Zahlungen im Rahmen von Spekulationsgeschäften. Sofern auch diese Voraussetzungen nicht gegeben sind, kommt ggf. noch eine Berufung darauf in Betracht, dass die Zahlungen nicht der Umsetzung eines Sanierungskonzeptes dienten. Dafür liegt die Messlatte allerdings sehr hoch, da der Gesetzgeber keine besonderen Anforderungen an dieses Sanierungskonzept gestellt hat.

\section{Wechselwirkungen zum Insolvenzanfechtungsrecht}

Letztlich sind auch noch Wechselwirkungen zu berücksichtigen. Insbesondere ist das Insolvenzanfechtungsrecht gem. $\$ \mathbb{S} 129 \mathrm{ff}$. InsO im Blick zu halten. Nach $\ 2$ Abs. 1 Nr. 4 COVInsAG sind zwar sämtliche kongruenten Deckungen nicht mehr anfechtbar sowie eine Reihe von inkongruenten Deckungen. Dies gilt allerdings nicht, wenn dem anderen Teil bekannt war, dass die Sanierungs- und Finanzierungsbemühungen des Schuldners nicht zur Beseitigung einer eingetretenen Zahlungsunfähigkeit geeignet waren. Darüber hinaus sind auch Anfechtungen von unentgeltlichen Leistungen gem. $\mathbb{S} 134$ InsO sowie Anfechtungen gegenüber Gesellschaftern gem. $\$ 135$ InsO weiterhin anfechtbar, soweit nicht eine Anfechtung - gegenüber Gesellschaftern, gem. $\$ 2$ Abs. 1 Nr. 2 COVInsAG - ausgeschlossen ist.

In dem Umfang, in dem der Insolvenzverwalter Zahlungen angefochten hat, die auch gegen die Massesicherungspflicht verstoßen haben, scheidet ein Anspruch gegen den Geschäftsführer aus.

\section{Wechselwirkungen zum Haftungs- und Deliktsrecht}

Die Haftung gem. $\$ 43$ GmbHG bleibt bestehen; gem. $\$ 43$ Abs. 2 GmbHG haften Geschäftsführer, welche ihre Obliegenheiten verletzen, der Gesellschaft für den entstandenen Schaden. Dazu gehört es auch, Vertragsangebote sachgerecht zu kalkulieren ${ }^{16}$ oder, sofern sich aus Unterlagen eines beauftragten Wirtschaftsprüfers bzw. Hinweisen des Betriebsrates ergibt,

16 So BGH, Urteil vom 28. Oktober 1971 - II ZR 49/70, NJW 1972, $154 \mathrm{f}$. = DB $1971,2353 \mathrm{f}$. 
dass die Voraussetzungen für die Beanspruchung von Kurzarbeitergeld vorliegen, dieses zu beantragen ${ }^{17}$.

Schließlich sind auch die Fälle der Haftung aus Delikt gem. $\$ 823$ Abs. 2 BGB i. V. m. Schutzgesetz zu berücksichtigen, die jedenfalls in den Fällen greifen, in denen nicht gerade $₫ 15$ a InsO oder $₫ 42$ Abs. 2 BGB das Schutzgesetz ist ${ }^{18}$.

\section{E. Fazit und Ausblick}

Alle diese Ausführungen zeigen, dass der Geschäftsführer trotz COVInsAG eine Reihe von unterschiedlichen Haftungsrisiken zu gegenwärtigen hat, insbesondere wenn die Insolvenzreife nicht auf den Folgen der COVID-19Pandemie beruht oder keine Aussichten darauf bestehen, eine bestehende Zahlungsunfähigkeit zu beseitigen. Darüber hinaus hat der Geschäftsleiter im Rahmen von $\$ 64$ Satz 2 GmbHG den Nachweis zu erbringen, dass die Zahlungen im ordnungsgemäßen Geschäftsgang erfolgten, was z. B. dann gegeben ist, wenn die Zahlungen der Aufrechterhaltung oder Wiederaufnahme des Geschäftsbetriebes oder der Umsetzung eines Sanierungskonzeptes dienen.

Geschäftsführer sind daher gut beraten, sowohl die Zahlungsfähigkeit zum 31. Dezember 2019 gründlich zu dokumentieren als auch hinsichtlich aller Zahlungen, die sie leisten, sicherzustellen, dass auch später nachgewiesen werden kann, dass diese im ordnungsgemäßen Geschäftsgang erfolgten ${ }^{19}$.

17 So BGH, Urteil vom 4. November 2002 - II ZR 224/00, BGHZ 152, $281 \mathrm{ff}$. = ZIP 2002, 2314 ff.; J. M. Schmittmann, Haftung der Organe in Krise und Insolvenz, 2. Auflage, Köln, 2018, Rdnr. 237.

18 Vgl. im Einzelnen: J. M. Schmittmann, Haftung der Organe in Krise und Insolvenz, 2. Auflage, Köln, 2018, Rdnr. 522 ff.

19 So J. M. Schmittmann, Die insolvenzrechtliche Komponente des Gesetzes zur Abmilderung der Folgen der COVID-19-Pandemie im Zivil-, Insolvenz- und Strafverfahrensrecht vom 27. März 2020, ZRI 2020, 234 (236). 